\section{A study of autopsy procedures in Ghana: implications for the use of autopsy data in epidemiological analyses}

\author{
Julius N. Fobil, ${ }^{1,2,3}$ Robert Kumoji, ${ }^{4}$ \\ Henry B. Armah, ${ }^{4,5}$ Eunice Aryee, ${ }^{1}$ \\ Francis Bilson, ${ }^{6}$ Derick Carboo, ${ }^{7}$ Frederick \\ K. Rodrigues, ${ }^{8}$ Christian G. Meyer, ${ }^{2}$ \\ Juergen May, ${ }^{2}$ Alexander Kraemer ${ }^{3}$ \\ 'School of Public Health, College of \\ Health Sciences, University of Ghana, \\ Legon, Accra, Ghana; \\ ${ }^{2}$ Bernhard Nocht Institute for Tropical \\ Medicine, Hamburg, Germany; \\ ${ }^{3}$ Department of Public Health Medicine, \\ School of Public Health, University \\ of Bielefeld, Bielefeld, Germany; \\ ${ }^{4}$ Department of Pathology, Korle-Bu \\ Teaching Hospital, Accra, Ghana; \\ ${ }^{5}$ Department of Pathology, University \\ of Pittsburgh Medical Center, Pittsburgh, \\ Pennsylvania, USA; \\ ${ }^{\circ 37}$ Military Teaching Hospital, Health \\ Division, Ministry of Defence (MOD), \\ Accra, Ghana; \\ ${ }^{7}$ Department of Chemistry, University \\ of Ghana and \\ ${ }^{8}$ Department of Biochemistry, University \\ of Ghana, Legon, Accra, Ghana
}

\section{Abstract}

The study of cause of death certification remains a largely neglected field in many developing countries, including Ghana. Yet, mortality information is crucial for establishing mortality patterns over time and for estimating mortality attributed to specific causes. In Ghana, autopsies remain the appropriate option for determining the cause of deaths occurring in homes and those occurring within 48 hours after admission into health facilities. Although these organ-based autopsies may generate convincing results and are considered the gold standard tools for ascertainments of causes of death, procedural and practical constraints could limit the extent to which autopsy results can be accepted and/or trusted. The objective of our study was to identify and characterise the procedural and practical constraints as well as to assess their potential effects on autopsy outcomes in Ghana. We interviewed 10 Ghanaian pathologists and collected and evaluated procedural manuals and operational procedures for the conduct of autopsies. A characterisation of the operational constraints and the Delphi analysis of their potential influence on the quality of mortality data led to a quantification of the validity threats as moderate (average expert panel score $=1$ ) in the generality of the autopsy operations in Ghana. On the basis of the impressions of the expert panel, it was concluded that mortality data generated from autopsies in urban settings in Ghana were of sufficiently high quality to guarantee valid use in health analysis.

\section{Introduction}

Misclassification is a major problem in cause of death ascertainment and presents an enormous threat to mortality data quality. ${ }^{1-3}$ For cause of death ascertainment, autopsies are the appropriate tools and play a significant role in the investigation of emerging health threats. ${ }^{1,2,4}$ However, the manner in which the autopsies are conducted is crucial for the validity of mortality data derived from them. ${ }^{3,5,6}$ Hence, there is the need for organ-based autopsy procedures to be investigated and carefully scrutinised for procedural limitations that may compromise the validity of autopsy results. ${ }^{7-9}$ For example, the lack of systematic studies and documentation on how organbased autopsy procedures are organised and delivered in Ghana offers no opportunity to capture the range of quality issues and validity threats surrounding mortality data generated in health facilities. ${ }^{1-3,6}$

Generally, clinical and laboratory diagnoses remain the commonest tools for cause of death ascertainment and when they fail to establish a cause then autopsy procedures are recommended.,3,6,10-12 Other less frequently used methods for ascertaining cause of death include verbal autopsies, which are often subject to strong criticisms on account of perceived and more or less obvious limitations. ${ }^{4,9,13,14}$ Some of the limitations of verbal autopsy methods based on validation studies are that they have low sensitivity and moderate specificity for identifying certain causes of deaths. 1,2,7,8,15 Thus, verbal autopsies apparently tend to misclassify or miss some deaths $s^{8,9}$ and consequently contribute to false estimates of cause-specific mortality.

Nonetheless, autopsies are widely used complementarily with clinical determinations as strategies to improve the accuracy of cause of death ascertainment in Ghana. On average, the Department of Pathology at Korle-Bu Teaching Hospital (KBTH) in Accra alone conducts approximately 5,000 autopsies annually, the vast majority (75\%) of which is referred for autopsy by the Coroner (personal communication). These coroner-referred deaths are often those occurring outside health facilities with
Correspondence: Julius N. Fobil, Bernhard Nocht Institute for Tropical Medicine, Bernhard Nocht Str. 74, 20359 Hamburg, Germany.

Tel. +49.40 .42818 .503 - Fax: +49.40 .42818 .512 .

E-mail: fobil@bni-hamburg.de

Key words: Ghana, health facility deaths, organbased examination, autopsies, validity threats, mortality, medical cause of death.

Contributions: conception and design were done by JFN, CGM, JM, AK; data and material gathering by JNF, EA, DC, FKR, HBA, RK, FB; synthesis, drafting and editing of the manuscript by all the authors.

Acknowledgements: funding for this study was generously provided by the Government of Ghana, through the GetFund Scholarship. The authors are grateful to all the pathologists who responded to our questionnaire.

Conflict of interest: the authors report no conflicts of interest.

Received for publication: 3 Septembe 2010. Accepted for publication: 20 January 2011.

This work is licensed under a Creative Commons Attribution 3.0 License (by-nc 3.0).

(C) Copyright J.N. Fobil et al., 2011

Licensee PAGEPress, Italy

Journal of Public Health in Africa 2011; 2:e7 doi:10.4081/jphia.2011.e7

no clinical history $6,16,17$ and the only way to ascertain the cause of death is then by autopsy. ${ }^{18,19}$ Therefore, organ-based autopsies are common procedures in Ghana, especially in the urban areas. ${ }^{20-24}$

However, despite their wide application in Ghana, no investigation has been conducted on how autopsies are conducted, what procedures are followed and what the limitations are. ${ }^{9}$ Moreover, neither is much known about the strategies employed for data quality assurance during routine post-mortems and/or autopsies nor whether standard operation procedures (SOPs) exist and whether physicians adhere to them. Given the relevance of cause of death information in national health policy planning, it is essential to investigate the certification processes in Ghana so that a review of procedures may be suggested and strategies developed to minimise validity threats associated with data generated by these procedures. This would significantly improve the validity and reliability of routine mortality records for use in scientific and epidemiological analyses as may be required. ${ }^{2,25,26}$

The aim of our study was to investigate and document the various procedures followed during cause of death ascertainment and reporting so as to assess the limitations of these procedures. 


\section{Materials and Methods}

We undertook a literature search and reviewed and analysed autopsy protocols and procedural manuals archived at the Official Repositories. We also contacted the 14 pathologists in Ghana via e-mail, explaining our intention to recruit them into this study, and requested indication of willingness to participate by e-mail replies. Twelve practising pathologists expressed willingness to participate in the study. We then e-mailed the questionnaire to them, which asked questions relating to procedural, organisational and practical steps involved in the conduct of autopsies in Ghana. Ten pathologists completed these and e-mailed back their responses. We checked the questionnaires for completeness and consistency. We followed up with personal and verbal interviews for clarification of issues and additional information based on new insights arising from the evaluation of the responses received. At the time this study was conducted (September 2007 to March 2008), the 14 practising pathologists were distributed as follows: ten in the Korle-Bu Teaching Hospital and University of Ghana Medical School, Accra; two in the Military Teaching Hospital, Accra; two in the Komfo Anokye Teaching Hospital and University Hospital, Kwame Nkrumah University of Science and Technology, Kumasi.

The responses were organised into the following themes: i) protocol and guidelines considerations, ii) organisational and practical arrangements, and iii) autopsy procedure and procedural issues.

An independent panel of experts conducted an audit, assessment and evaluation of the responses according to the three themes, focusing on the features that could potentially lead to misclassification. The panel of experts was composed of five expert pathologists independently constituted by the study team at the start of the study. The criteria for the audit, assessment and evaluation were based on the extent of potential process flaws associated with each theme and the rationale for validity threat characterisation were based on the number of process flaws (Table 1). For each theme, the panel scores were aggregated (cumulative score) and the average panel score computed. An average score of zero (0) represented no validity threat, an average score of one (1) represented a moderate validity threat and an average score of two (2) meant a severe validity threat.

\section{Results}

This section presents the results of the Delphi analysis, the evaluation of clinical pro- tocols and synthesis of e-mail responses from pathologists in Ghana.

Table 2 shows a summary of the expert assessment and the corresponding weight of validity threat associated with each process. We observed some methodological limitations in the autopsy process according to the Delphi analysis. In particular, the limitation imposed by the lack of accurate instruments presented a moderate validity threat (score $=1$ ) to the quality of data generated from clinical diagnoses as per expert panel conclusions (Table $2)$. While protocol and guidelines considerations received an average panel score of (0.8), implying a contribution of moderate threat, both autopsy procedure and surgical infrastructure/instrumentation were assigned an average score of (0.6), representing a borderline case between no validity threat and moderate validity threat.

Analysis of policy documents and personal interviews with pathologists revealed that there were no laid down formal protocols and/or guidelines for conducting cause of death ascertainment in Ghana. However, a standard system of practice, which had evolved from several years of regular practice, existed. For instance, there were autopsy request forms on which to record details of required autopsy information. In addition, although the International Classification of Diseases (ICD) system was not in regular use, pathologists and physicians were definitely aware of its existence ICD (personal communication).

In general, two major methodological systems were identified, namely pre-mortem and post-mortem procedures for cause of death certification in Ghana. The two evolved as legally established systems that complement each other. We observed the existence of other practices that were both unacceptable and, without doubt, illegal. These practices were characterised as certification without autopsies and outright burial without ascertainment of cause and were reported to be common in normal practice. In the Ghanaian context, the descriptions of each type of cause of death certification system with reference to the accur acy, limitation, coverage and the ultimate

Table 1. Panel guide.

\begin{tabular}{lcl} 
Exxtent of flaws & Score & Characterisation \\
No perceived flaw & 0 & No validity threat \\
One flaw & 1 & Moderate validity threat \\
\hline Two or more flaws & 2 & Severe validity threat \\
\hline
\end{tabular}

impact on the quality of cause of mortality data are provided below.

\section{Pre-mortems (clinical diagnoses)}

Pre-mortem procedures were reported to include the routine clinical and laboratory investigations applied to inpatients and outpatients. In establishing a definitive cause of a clinical condition, presumptive diagnoses would be made based on verbal questioning and laboratory investigations at health facilities on condition that such clinical and laboratory procedures would establish a definitive cause of the clinical condition. In that circumstance, the attending physician would write and assign a clinical cause. On the contrary, if clinical and laboratory investigations failed to establish a definitive cause before death, then post-mortem procedures would be applied until the cause of death was determined and a certificate issued. Cause of death certificates are requirements for securing burial permits. The Vital Registration System (VRS) therefore has registration sites (subsidiary offices) within the premises of all mortuaries. At these registration sites, registration officials collate all certificates of cause of death and issue burial permits in order to permit burials in controlled cemeteries.

\section{Post-mortems (autopsies)}

Post-mortems were categorised as a constellation of routine procedures conducted by qualified pathologists. These procedures broadly encompassed all categories of organ examination autopsies. Additionally, certification without proper clinical investigation, assignment of cause of death by unqualified persons, outright burials without cause of death certificates, etc. were also classified under postmortems. It was revealed in the interviews that

Table 2. Summary of expert panel scores.

\begin{tabular}{lccc} 
Source of data flaws & $\begin{array}{c}\text { Cumulative } \\
\text { score }\end{array}$ & $\begin{array}{c}\text { Average } \\
\text { score }\end{array}$ & $\begin{array}{l}\text { Validity } \\
\text { threat }\end{array}$ \\
Protocol and guidelines considerations & 4 & 0.8 & Moderate \\
Organisational and practical arrangements & 5 & 1.0 & Moderate \\
\hline Autopsy procedure & 3 & 0.6 & Moderate \\
Surgical infrastructure/instrumentation & 3 & 0.6 & Moderate \\
\hline Organ conditions & 5 & 1.0 & Moderate \\
Personal characteristics of deceased & 6 & 1.2 & Moderate \\
\hline Unacceptable/illegal cause of death certificationsto severe & 7 & 1.4 & Moderate \\
\hline
\end{tabular}


the laws establishing the VRS forbade burials without certificate and/or certificates without cause of death. However, some respondents reported that such practices did occur occasionally and generally were believed to be more pronounced in areas/regions with low literacy.

\section{Dissection-based organ-examin- ation autopsies in Ghana}

Organ-examination autopsies were defined as the anatomical and organ inspection procedures that allowed for the establishment of cause of death. These procedures were reported to be standard and required a thorough inspection of organ-state. Autopsies, by Ghanaian law, were mandatory for all deaths without established cause, especially deaths occurring in homes or outside health facilities. Such deaths were classified in the national statutes as Coroner's cases. We identified the following as conditions necessitating the conduct of organ-based autopsies: i) unconcluded or unconfirmed pre-mortems, ii) deaths occurring within 24 hours after admission into a health facility, and iii) deaths at homes or outside a health facility (Coroner's cases).

During the interviews, some pathologists reported that all deaths occurring in health facilities required assignment of cause of death certificates. However, owing to practical constraints, some deaths escaped autopsy processes and reportedly went for burial without cause of death ascertainment, especially in rural areas of Ghana (personal communication).

The autopsy process was examined at three levels of activity, namely: i) whether the process took medical practice protocols into consideration, ii) the organisational and practical arrangements considered in the process, and iii) the technical procedures applied. There were autopsy protocols and guidelines for the conduct of autopsies and consultation of these protocols were mandatory before commencement of autopsies. In some instances, discretionary actions were taken especially regarding what tissues to sample for histological examination or when to do microbiological and toxicological tests. Although exercise of discretion was envisaged as a potential source of validity threat to the autopsy and cause of death certification process, the Delphi analysis essentially revealed inconsequential validity threats. The reason was that autopsy findings of any unusual or atypical cases were presented to colleagues by the pathologist in charge at weekly review conferences. Sampling additional tissues or additional testing was conducted if that was the consensus after the review. The additional sampling/testing would potentially eliminate or at least minimise the frequency of systematic biases or misclassification owing to exercise of discretion.
The organizational and practical arrangements were classified as the preparatory works before commencement of an autopsy. These included review of clinical records, laboratory tests and any other relevant information, including verbal autopsies, related to the case prior to the autopsy. This was meant to inform what special techniques, precautions, tissues to be sampled for histological examination and what microbiological or toxicological tests were required. These preparations differed among corpses because the findings of some of the reviews could be so similar to previously reviewed clinical notes that there was no need for any special techniques, precautions and tissue sampling, microbiological or toxicological testing procedures.

\section{The local context}

In Ghana, the standard Rokitansky technique was reported as the main procedure in autopsies. This technique involves an anterior midline incision from neck to perineum with enbloc removal of internal organs for subsequent systematic dissection. Incision with reflection of the scalp and sawing of the skull to remove and examine the brain in some cases was reportedly a routine procedure. However, it was noted that minor variations in the application of the standard technique to different corpses existed, depending on the particular pathological findings anticipated. For example, the protocol stipulated a dissection of the heart and lungs together if the anticipated finding related to a congenital heart disease, rather than the usual separate dissection of the heart and lungs. These considerations were reported as usually standardised protocols and hence did not affect the final results. In the event that the technique did not result in conclusive findings after additional histological, microbiological and toxicological testing, the cause of death was reported to be designated as undetermined or undeterminable.

\section{Unacceptable/illegal cause of death certifications}

Instances where physicians took bribes and assigned an uninvestigated cause of death for various reasons were reported. This practice was regarded as unacceptable, and to ensure that this type of irregularity did not happen, the vetting of cause of death certificates policy was proposed and implemented in KBTH. In other circumstances, it was reported that physicians, under compulsion or inducement, assigned cause of death certificates without autopsies. Although deemed illegal under Ghanaian law, factors that dictated such circumstances were reported to include pressures from relatives of deceased persons, who demanded the release of the bodies for imme- diate burial. Despite claims that such abnormalities rarely occurred, the irregularities certainly remained an important issue as far as the validity of cause of death ascertainment was concerned. This study could not determine the actual rate and extent of the irregularities as it was difficult to differentiate between valid and invalid records, once they were deposited at the repository of the VRS.

Outright burials without cause of death ascertainment were reported to be common in informal settlements where the law was not properly enforced, allowing for most burials to go unchecked through bribery at the cemeteries. Although this limitation was believed to be extensive in rural settings and almost improbable in highly urbanised areas, the Delphi analysis placed it on moderate validity threat in highly urbanised settings (score 1) and severe threat (score 2) in rural settings.

An assessment of all the processes in the conduct of autopsies in Ghana showed that checks were in place to ensure high professional standards, although assurance of best practice did not guarantee complete elimination of biases owing to misclassification. In view of the strict adherence to best practice as observed in Ghana, systematic errors such as deferential misclassification would be avoided and, despite the moderate validity threats envisaged by the expert panel assessment, such biases would be those of non-deferential errors that are inherent in most standard clinical techniques including the gold standard laboratory methods.

\section{Discussion}

During dissections, if an organ detail required the use of a highly specific medical tool, the accuracy limits of such clinical equipment ultimately determined the accuracy of the test results. Moreover, many cause-specific clinical conditions were observed to be difficult to determine from mere organ conditions, for example, metabolic causes were hard to determine from organ inspection unless pre-empted in the clinical procedures. As per expert panel deliberations, this limitation presented moderate validity ( score $=1$ ) threat to data obtained from autopsy procedures. This meant that cause of death data from autopsies would be skewed because many clinical conditions did not manifest in organ structure and were likely to be difficult to determine. Limitations imposed by difficulties in determining personal characteristics of the deceased that had bearing on cause-specific clinical conditions were of a major concern to the panel of experts. For example, personal characteristics such as age of individuals were hard to determine if unknown, and it was observed that 
most people did not know their ages and were likely to guess their ages to the nearest whole number in a convincing arbitrary manner (e.g. 29 years, 31 years, 41 years, etc.), giving a false impression of their ages. This limitation received an average expert panel score (1.2) implying it presented a moderate validity threat to data derived from autopsies.

\section{Conclusions}

Potential validity threats, associated with autopsy processes in Ghana according to the Delphi analysis, were of no or limited consequence on the quality of output data. Organizational and practical constraints were rated to present moderate validity threats to output data. Severe threats were associated with the protocol and guideline considerations in rural areas compared to moderate threats in urban settings.

Indeed, the quality of data resulting from cause of death reporting would largely be influenced by the culture of practice and the robustness of the reporting systems in place. The current culture of reporting on vital events may be fraught with imperfections. Nevertheless, substantial improvements in the strength, robustness and reliability of the reporting systems may become achievable only if developed hand-in-hand with existing health information strategies at the national and district levels. Concomitant improvements of governance structures and an agenda for social research and development monitoring on health information systems could strengthen the endeavours made in the reporting system. ${ }^{25,27-30}$ For a reporting system to be useful, the data it generates must be internally consistent, plausible and reflect epidemiological characteristics at the community-level data. ${ }^{25}$ On the basis of the Delphi analyses, it was concluded that mortality data generated from autopsy findings in urban settings in Ghana were of adequate quality for use in health analysis. Nonetheless, the reporting infrastructure at its current strength did not guarantee adequate reliability of mortality data from rural settings in Ghana.

\section{References}

1. Quigley MA, Chandramohan D, Rodrigues LC. Diagnostic accuracy of physician review, expert algorithms and data-derived algorithms in adult verbal autopsies. Int $\mathbf{J}$ Epidemiol 1999;28:1081-7.

2. Quigley MA, Chandramohan D, Setel P,
Binka F, Rodrigues LC. Validity of dataderived algorithms for ascertaining causes of adult death in two African sites using verbal autopsy. Trop Med Int Health 2000; 5:33-9.

3. Tettey Y, Wiredu EK. Autopsy studies on still births in Korle Bu Teaching Hospital: pathological findings in still births and their placentae. West Afr J Med 1997;16: 12-19.

4. Boulle A, Chandramohan D, Weller P. A case study of using artificial neural networks for classifying cause of death from verbal autopsy. Int J Epidemiol 2001;30: 515-20.

5. Wiredu EK, Armah HB. Cancer mortality patterns in Ghana: a 10-year review of autopsies and hospital mortality. BMC Public Health 2006;6:159.

6. Wiredu EK, Tettey Y. Autopsy studies on still births in Korle Bu Teaching Hospital. II: Causes of death in 93 still births. West Afr J Med 1998;17:148-52.

7. Chandramohan D, Maude GH, Rodrigues LC, Hayes RJ. Verbal autopsies for adult deaths: their development and validation in a multicentre study. Trop Med Int Health 1998;3:436-46.

8. Chandramohan D, Rodrigues LC, Maude GH, Hayes RJ. The validity of verbal autopsies for assessing the causes of institutional maternal death. Stud Fam Plann 1998;29:414-22.

9. Chandramohan D, Setel P, Quigley M. Effect of misclassification of causes of death in verbal autopsy: can it be adjusted? Int J Epidemiol 2001;30:509-14.

10. Wiredu EK, Kumoji R, Seshadri R, Biritwum RB. Osteometric analysis of sexual dimorphism in the sternal end of the rib in a West African population. J Forensic Sci 1999;44:921-5.

11. Wiredu EK, Nyame PK. Stroke-related mortality at Korle Bu Teaching Hospital, Accra, Ghana. East Afr Med J 2001;78:180-4.

12. Wolf-Gould C, Osei L, Commey JO, Bia FJ. Pediatric cerebral malaria in Accra, Ghana. J Trop Pediatr 1992;38:290-4.

13. Mills S, Williams JE, Wak G, Hodgson A. Maternal mortality decline in the KassenaNankana district of northern Ghana. Matern Child Health J 2008;12:577-85.

14. Gyapong J0, Chinbuah MA, Gyapong M. Inadvertent exposure of pregnant women to ivermectin and albendazole during mass drug administration for lymphatic filariasis. Trop Med Int Health 2003;8:1093-101.

15. Allotey P, Reidpath D. Establishing the causes of childhood mortality in Ghana: the 'spirit child'. Soc Sci Med 2001;
52:1007-12.

16. Armah H, Dodoo AK, Wiredu EK, et al. High-level cerebellar expression of cytokines and adhesion molecules in fatal, paediatric, cerebral malaria. Ann Trop Med Parasitol 2005;99:629-47.

17. Armah HB, Wilson NO, Sarfo BY, et al. Cerebrospinal fluid and serum biomarkers of cerebral malaria mortality in Ghanaian children. Malar J 2007;6:147.

18. Anim JT. Cerebral macroaneurysms in a Ghanaian adult population. An autopsy study. Atherosclerosis 1985;54:37-42.

19. Anim JT. Pattern of atherosclerosis in a Ghanaian adult population: an autopsy study. Afr J Med Med Sci 1987;16:203-12.

20. Aleksenko L, Gyasi R. Hypoglycaemia in an infant of a mother with fasting hyperglycaemia - a case report. Ghana Med J 2005;39:108-11.

21. Apeagyei F, Lamplugh SM, Hendrickse RG, Affram K, Lucas S. Aflatoxins in the livers of children with kwashiorkor in Ghana. Trop Geogr Med 1986;38:273-6.

22. Clegg-Lamptey JN, Armah H, Naaeder SB, Adu-Aryee NA. Position and susceptibility to inflammation of vermiform appendix in Accra, Ghana. East Afr Med J 2006;83:6703.

23. Gyasi R, Tettey Y. Childhood deaths from malignant neoplasms in accra. Ghana Med J 2007;41:78-81.

24. Matekole M, Affram K, Lee SJ, et al. Hypertension and end-stage renal failure in tropical Africa. J Hum Hypertens 1993;7:443-6.

25. Setel PW, Sankoh O, Rao C, et al. Sample registration of vital events with verbal autopsy: a renewed commitment to measuring and monitoring vital statistics. Bull World Health Organ 2005;83:611-7.

26. Timaeus I, Graham W. Measuring adult mortality in developing countries: a review and assessment of methods. 1998 (unpublished typescript).

27. Court C. WHO claims maternal mortality has been underestimated. BMJ 1996;312:398.

28. de Beer M, Padayachee GN, Ijsselmuiden C, Coetzee D. The reliability of mortality data in Johannesburg. S Afr Med J 1993;83:597-601.

29. Mantel GD, Moodley J. Can a developed country's maternal mortality review be used as the 'gold standard' for a developing country? Eur J Obstet Gynecol Reprod Biol 2002;100:189-95.

30. Wiley AS, Pike IL. An alternative method for assessing early mortality in contemporary populations. Am J Phys Anthropol 1998;107:315-30. 